\title{
A Simple Method for Safer Flexible Fiberoptic Laryngoscopy in the Era of COVID-19
}

\author{
Christopher A. Maroun $^{1} \cdot$ Daniel S. Faddoul ${ }^{1} \cdot \operatorname{Roger}$ V. Moukarbel $^{1}$
}

Received: 2 September 2020/ Accepted: 19 October 2020/Published online: 23 October 2020

(C) Association of Otolaryngologists of India 2020

The 2019 novel coronavirus (SARS-CoV-2) has had tremendous impact on healthcare systems around the globe, resulting in a shift in the distribution of resources and a rapid change in the provision of care. Otolaryngologists in particular are at increased risk of contact with respiratory secretions and subsequent infection, therefore efforts to reduce exposure through prioritization of procedures and utilization of personal protective equipment (PPE) have been widely employed [1]. While in many institutions it is agreed upon that a number of instances of clinical contact can be safely deferred until a later time, there are cases, particularly within the cancer population, where this may not be a viable option. For this reason, it is important to consider additional measures to protect the provider during necessary clinical encounters so that a lapse in patient care can be avoided, without compromising safety.

In the evaluation of the cancer patient, a key element of assessment is a thorough evaluation of the aerodigestive tract. For the modern head and neck surgeon, one of the most useful tools for visualization is the flexible laryngoscope. However, unnecessary flexible laryngoscopy in the era of COVID-19 is generally being avoided due to the increased risk of transmission of viral particles to the otolaryngologist [1]. In fact, it has been reported that exposure to aerosolized viral particles may be highest during manipulation of the aerodigestive tract, such as

\section{Roger V. Moukarbel}

rm17@aub.edu.lb

1 Department of Otolaryngology Head and Neck Surgery, American University of Beirut Medical Center, 6th Floor, Hamra, Beirut 1107 2020, Lebanon during intubation or other minimally invasive procedures [2].

Flexible laryngoscopy in particular poses an additional risk due to the potential for coughing and sneezing during manipulation. This may increase the likelihood of contracting illness from viral carriers, as moderate viral loads have been found within the respiratory tracts of infected patients, even when asymptomatic [3]. There have been several recommendations that advocate heightened caution during airway procedures in order to minimize the risk of transmission to the provider [2, 4]. However, there have been no official recommendations for reducing viral transmission during flexible laryngoscopy in particular [5].

Recently in our practice, similar to reports from other institutions, preparation for flexible laryngoscopy has entailed a comprehensive donning of PPE, including: surgical gown, bouffant cap, eye protection, face shield, N95 mask with overlying surgical mask, and two pairs of latex gloves (Fig. 1a). However, in addition to these measures, we have employed an additional layer of protection specifically targeting the risks inherent to flexible laryngoscopy. A properly fitted N95 mask is placed on the patient, and the laryngoscope is introduced through a pinpoint aperture made with scissors within the mask, into the nasal or oral cavity (Fig. 1a-c). This provides a barrier to viral droplets that may be ejected during the procedure, without sacrificing mobility or ease of operation on the otolaryngologist's behalf. The mask will capture the droplets and reduce the exposure.

The advantage of this method is that it is simple and accessible to all practitioners. The cost of the mask is negligible compared to the benefit. We advocate the use of a disposable N95 mask without valve. It is readily available in most centers and even private clinics. The aperture created with the scissors should be just large enough to introduce the scope. The patient should keep the mask on 


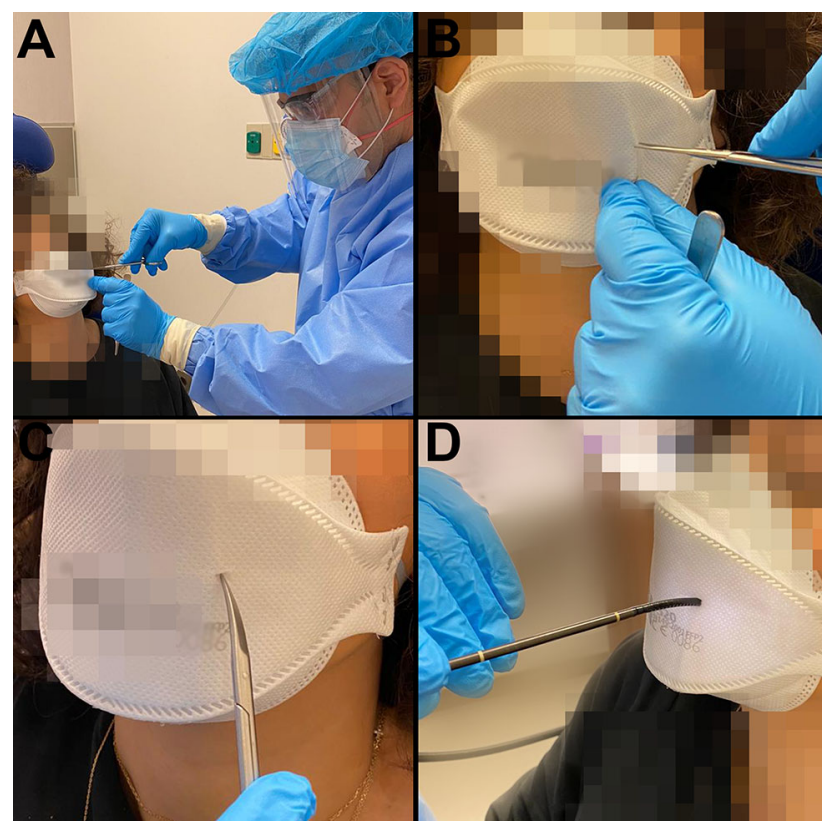

Fig. 1 Procedure for implementing precautions during laryngoscopy. a Surgeon dressed in full PPE during the procedure. $\mathbf{b}$ Creation of the mask aperture using scissors. $\mathbf{c}$ Demonstration of the pinpoint size of the aperture. d Using the laryngoscope through the newly created aperture

throughout the procedure, as well as afterwards, and he or she can leave the premises with the mask on.

The current state of the pandemic requires that we seize every opportunity to mitigate the risk of viral transmission to healthcare professionals, especially those inherently at higher risk. Here, we have proposed a very simple intervention that may provide plausible benefit with no added risk to the patient. Therefore, in cases where flexible laryngoscopy is required, we advocate the use of our method moving forward.

\section{Funding None.}

Compliance with ethical standards

Conflict of interest None.

\section{References}

1. Chan JYK, Wong EWY, Lam W (2019) Practical aspects of otolaryngologic clinical services during the 2019 novel coronavirus epidemic. JAMA Otolaryngol Head Neck Surg. https://doi.org/10.1001/jamaoto.2020.0488

2. Wax RS, Christian MD (2020) Practical recommendations for critical care and anesthesiology teams caring for novel coronavirus (2019-nCoV) patients. Can J Anesth. https://doi.org/10.1007/s12630-020-01591-x

3. Zou L, Ruan F, Huang M et al (2020) SARS-CoV-2 viral load in upper respiratory specimens of infected patients. NEJM 382:1177-1179

4. Luo M, Cao S, Wei L et al (2020) Precautions for intubating patients with COVID-19. Anesthesiology. https://doi.org/10.1097/ALN.0000000000003288

5. Vukkadala N, Qian J, Holsinger C, Patel ZM, Rosenthal E (2020) COVID-19 and the otolaryngologist: preliminary evidence-based review. Laryngoscope. https://doi.org/10.1002/lary.28672

Publisher's Note Springer Nature remains neutral with regard to jurisdictional claims in published maps and institutional affiliations. 